\title{
Ibrutinib reduces neutrophil infiltration, preserves neural tissue and enhances locomotor recovery in mouse contusion model of spinal cord injury
}

\author{
Somayyeh Torabi ${ }^{1}$, Seyed Hadi Anjamrooz ${ }^{1}$, Zahra Zeraatpisheh ${ }^{2}$, Hadi Aligholi ${ }^{2}$, Hassan Azari $^{3}$ \\ ${ }^{1}$ Department of Anatomical Sciences, School of Medicine, Shiraz University of Medical Sciences, Shiraz, ${ }^{2}$ Department of Neuroscience, School of \\ Advanced Medical Sciences and Technologies, Shiraz University of Medical Sciences, Shiraz, Iran, ${ }^{3}$ Department of Neurosurgery, McKnight Brain \\ Institute, University of Florida, Gainesville, FL, USA
}

\begin{abstract}
Following acute spinal cord injury (SCI), excessive recruitment of neutrophils can result in inflammation, neural tissue loss and exacerbation of neurological outcomes. Ibrutinib is a bruton's tyrosine kinase inhibitor in innate immune cells such as the neutrophils that diminishes their activation and influx to the site of injury. The present study evaluated the efficacy of ibrutinib administration in the acute phase of SCI on neural tissue preservation and locomotor recovery. Ibrutinib was delivered intravenously at $3.125 \mathrm{mg} / \mathrm{kg}$ either immediately, 12 hours after, or both immediately and 12 hours after SCI induction in adult male C57BL/6 mice. Neutrophil influx into the lesion area was evaluated 24 hours following SCI using light microscopy and immunohistochemistry methods. Animals' body weight changes were recorded, and their functional motor recovery was assessed based on the Basso mouse scale during 28 days after treatment. Finally, spinal cord lesion volume was estimated by an unbiased stereological method. While animals' weight in the control group started to increase one week after injury, it stayed unchanged in treatment groups. However, the double injection of ibrutinib led to a significantly lower body weight compared to the control group at 4 weeks post-injury. Mean neutrophil counts per visual field and the lesion volume were significantly decreased in all ibrutinib-treated groups. In addition, ibrutinib significantly improved locomotor functional recovery in all treated groups, especially in immediate and double-injection groups. Neural tissue protection and locomotor functional recovery suggest ibrutinib treatment as a potent immunotherapeutic intervention for traumatic SCI that warrants clinical testing.
\end{abstract}

Key words: Spinal cord injuries, Ibrutinib, Recovery of function

Received November 16, 2020; 1st Revised March 4, 2021; 2nd Revised April 26, 2021; Accepted May 10, 2021

\footnotetext{
Corresponding author:

Hassan Azari (10

Department of Neurosurgery, McKnight Brain Institute, University of Florida, Gainesville, FL 32611, USA

E-mail: hassan.azari@neurosurgery.ufl.edu Hadi Aligholi (iD

Department of Neuroscience, School of Advanced Medical Sciences and Technologies, Shiraz University of Medical Sciences, Shiraz 7134814336, Iran

E-mail:aligholi@sums.ac.ir
}

\section{Introduction}

Spinal cord injury (SCI) is a catastrophic neurological event that desperately needs therapeutic interventions primarily to control the inflammation which happens immediately after primary neural tissue insult. Excessive inflammation ultimately leads to neural tissue destruction, cavity formation, scarring, and subsequent sensory and motor disturbances. Despite decades of intensive research to establish

\section{Copyright ( $) 2021$. Anatomy \& Cell Biology}

This is an Open Access article distributed under the terms of the Creative Commons Attribution Non-Commercial License (http://creativecommons.org/licenses/by-nc/4.0/) which permits unrestricted non-commercial use, distribution, and reproduction in any medium, provided the original work is properly cited. 
effective therapeutic strategies in SCI, to date, no successful recuperation has yet been achieved $[1,2]$.

Following the primary damage to the spinal cord as a result of transection, compression, contusion or ischemic insults, a series of secondary injuries begin with inflammation. Inflammation acts as a double-edged sword and undisputedly may lead to further neurologic dysfunctions if not controlled [3-5]. Amongst white blood cells, neutrophils are the first defensive leukocytes that arrive at the site of inflammation. Despite their beneficial effects in recruiting phagocytic cells such as the macrophages to remove debris and resolving inflammation, an excessive number of neutrophils may lead to an imbalance and dysregulated milieu that accelerates neural tissue loss through the release of their oxidative and proteolytic enzymes [6, 7]. Studies have shown that the modulation of neutrophil infiltration into the site of neural injury presents a promising approach for the management of secondary events following SCI [8].

Ibrutinib, a Food and Drug Administration (FDA)-approved medication, can block Bruton's tyrosine kinase (BTK) in some immune cells such as B cells and myeloid cells [912]. Ibrutinib is primarily known for its essential role in the treatment of mantle cell lymphoma and chronic lymphocytic leukemia as well as other B cell malignancies $[9,13,14]$. In macrophages, BTK regulates the secretion of inflammatory cytokines upon stimulation [15]. BTK is also involved in neutrophil margination, adherence to and their crawling along the endothelium, and finally their migration into the damaged tissues [16]. Evidence indicates that activation of BTK increases caspase-1 in myeloid cells. This phenomenon in turn releases interleukin (IL)-1 $\beta$ to the extracellular space which can strongly induce more neutrophil recruitment and intensify inflammatory response. Ibrutinib irreversibly binds to a conserved cysteine residue of BTK, and thereby suppresses phosphorylation and activation of downstream signaling proteins in neutrophils to mitigate acute inflammation. Studies have also shown that BTK-inhibition could subside inflammation and tissue injury in the liver [17] and brain ischemia reperfusion models [18]. Moreover, the role of BTK-inhibition for regulating inflammation in acute lung injury and lethal flu has been established $[19,20]$.

The present study was aimed to evaluate the immunomodulatory effect of ibrutinib, a selective BTK-inhibitor, in terms of neutrophil infiltration into the injured spinal cord tissue and the subsequent effects on neural tissue preservation and functional motor recovery in a mouse contusion model of SCI. Furthermore, we investigated the best time course of drug administration in the acute phase of SCI.

\section{Materials and Methods}

\section{Animals and experimental design}

All procedures were performed according to the Shiraz University of Medical Sciences ethical committee guidelines (approval no. 15704). All mice were maintained in 12-hour/12-hour light/dark cycle with adequate food and water access. Attempts were made to alleviate the animals' distress as much as possible.

Sixty adult male C57BL/6 mice weighing 25-30 g were randomly divided into five experimental groups $(\mathrm{n}=12$ per group, Fig. 1). After surgery, animals either received $100 \mu \mathrm{l}$ of phosphate buffer saline (PBS) intravenously via tail vein immediately after injury (SCI/vehicle) or $100 \mu \mathrm{l}$ of ibrutinib (dissolved in $0.1 \mathrm{M} \mathrm{PBS}$ at $3.125 \mathrm{mg} / \mathrm{kg}$ ) [18] immediately after injury (SCI/immediate ibrutinib), 12 hours after injury (SCI/12-hour ibrutinib) and both immediately and 12 hours after injury (SCI/double injection ibrutinib). In sham control group, laminectomy procedure was performed without any injury to the spinal cord. Twenty-four hours after surgery, 5 mice from each group were randomly sacrificed to evaluate neutrophil infiltration into the injured spinal cord tissue.

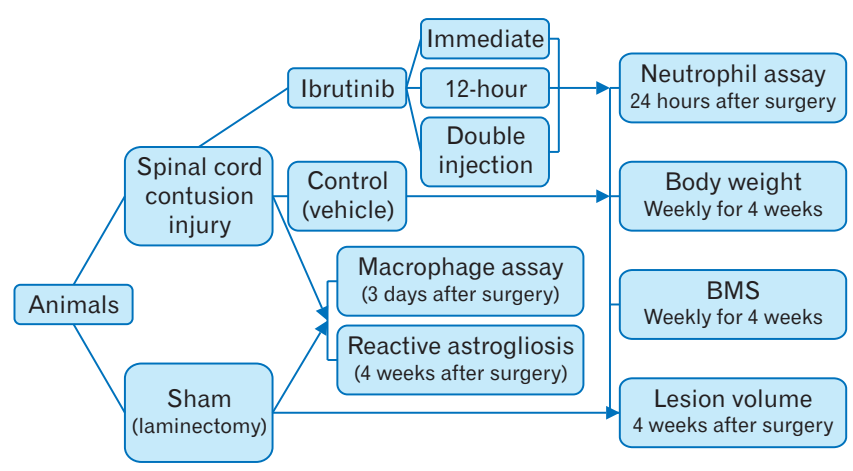

Fig. 1. Study design: animals were divided into sham (laminectomy only) and spinal cord injury (SCI) groups. After inducing SCI, animals were treated either with phosphate buffer saline (PBS) (Control) or ibrutinib in PBS; immediately, 12 hours and both immediately and 12 hours after injury (ibrutinib/immediate, ibrutinib/12-hour, ibrutinib/double injection). Neutrophil influx into the lesion area was analyzed 24 hours after injury. Body weight and locomotor function were evaluated every week for 4 weeks. To confirm injury to the spinal cord, immunofluorescent staining against ED1 and glial fibrillary acidic protein were performed at 3 days and 4 weeks after surgery, respectively. Lesion volume was estimated at the end of the study. BMS, Basso mouse scale. 


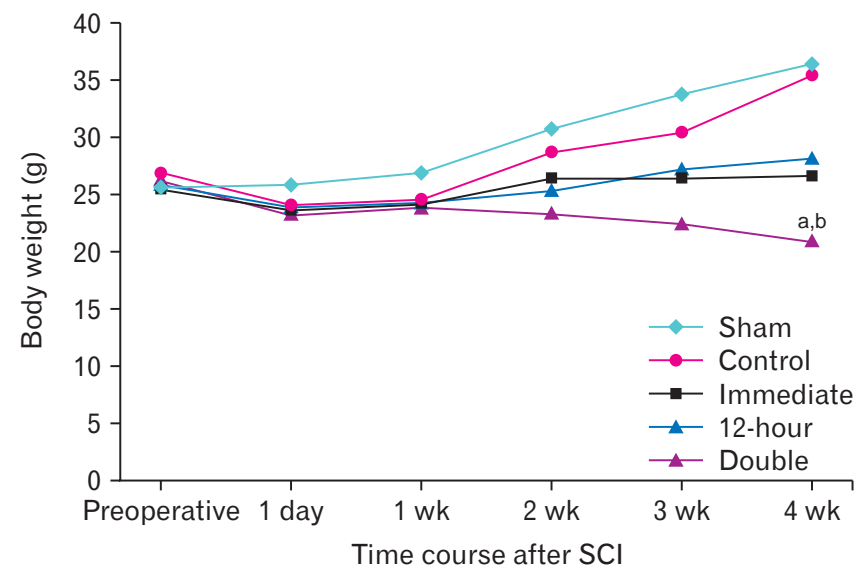

Fig. 2. Body weight changes during 4 weeks after SCI. Slight weight loss occurred in all SCI groups one week after injury. While the sham and control groups continued to gain weight starting at one week postinjury, it was plateaued in ibrutinib/immediate and ibrutinib/12-hour groups but significantly decreased in ibrutinib/double injection group at 4 weeks post-SCI. SCI, spinal cord injury. ${ }^{a} P<0.05$ vs. SCI/vehicle, ${ }^{b} P<0.05$ vs. Sham.

The remaining animals were evaluated in terms of locomotor recovery for 4 weeks. Finally, all mice were sacrificed, and the spinal cord lesion volume was assessed. The body weight was measured before surgery and then weekly for 4 weeks after surgery (Fig. 2).

\section{Induction of spinal cord injury}

For SCI surgeries, animals underwent a laminectomy at T10 level under deep anesthesia with an intraperitoneal injection of a mixture of $10 \mathrm{mg} / \mathrm{kg}$ xylazine hydrochloride and $90 \mathrm{mg} / \mathrm{kg}$ ketamine hydrochloride. Complete anesthesia was confirmed by testing response to paw-pinch reflex. To create a laminectomy, after shaving by an electric clipper and scrubbing the thoracolumbar skin with betadine solution and alcohol, a midline skin incision was made on the back of the animals. The paravertebral muscles were dissected away from spinous processes, and lamina was removed by using an electric dental drill instrument. To induce contusive SCI, an 8-gram bar-shaped weight with $27 \mathrm{~cm}$ length and a 1 $\mathrm{mm} \times 2 \mathrm{~mm}$ tip was applied for 10 minutes on the dura mater overlying the spinal cord [21]. Next, the paravertebral muscles, superficial fascia, and skin were sutured. For hydration, the animals received $1 \mathrm{ml}$ of $0.9 \%$ saline intraperitoneally. To prevent hypothermia, the animals were placed on a heating pad during surgery and recovery period. The mice bladder was manually expressed three times a day until spontaneous voiding returned. For analgesia, the mice received $1.5 \mathrm{mg} /$ $\mathrm{kg}$ of tramadol every 8 hours for 2 days. Moreover, for preventing urinary tract infection, $4 \mathrm{mg} / \mathrm{kg}$ of gentamicin was administrated once a day for 3 days.

\section{Tissue preparation for histological studies}

For neutrophil infiltration assay or lesion volume assessment, animals were anesthetized by an overdose of ketamine and xylazine 24-hour and 4 weeks after injury, respectively, and were perfused transcardially with $0.9 \%$ cold saline and then by $4 \%$ paraformaldehyde in $0.1 \mathrm{M}$ PBS. Subsequently, a piece of the vertebral column containing the lesion site was isolated and post fixed in $4 \%$ paraformaldehyde overnight. The spinal cord was accurately dissected from the column and was further processed and embedded in paraffin [22]. Next, $8 \mu \mathrm{m}$ serial cross sections were prepared using a microtome (MICROM HM 325, Walldorf, Germany) and sections were placed on superfrost plus glass slides with $50 \mu \mathrm{m}$ intervals.

\section{Neutrophil assay in spinal cord injury tissue}

After deparaffinization through xylenes and rehydration in a series of graded alcohols, we utilized Congo red-Hematoxylin staining on serial cross sections that were prepared 24-hour after SCI. The tissues were immersed in Hematoxylin for 10 minutes and were stained with $0.5 \%$ Congo red for 20 minutes. After clearing and cover-slipping, we counted the neutrophils in the SCI tissues by using of Nikon E-200 microscope (Nikon, Tokyo, Japan), connected to a computer with the Porsche's software. For neutrophil enumeration, we randomly chose three fields in each section in the first step and counted cells with unique features of neutrophils such as segmented ring nuclei and pale cytoplasm [23] by $60 \times$ oil immersion objective lens inside the injury site. Finally, the total sum of neutrophils in each section was calculated and the average number of neutrophils per visual field in each animal was determined.

\section{Immunohistochemical assessment of Ly6G, CD68 (ED1) and GFAP expression following spinal cord injury}

We utilized the anti-ly6G antibody as a surface marker of infiltrated neutrophils. Tissue sections from the rostral, caudal, and epicenter of the injured site were selected. After deparaffinization with xylene for 3 minutes, sections were hydrated in a series of graded alcohol. Later, the sections were incubated with sodium citrate buffer solution, $\mathrm{pH}$ 6.0, 
for 40 minutes at $95^{\circ} \mathrm{C}-100^{\circ} \mathrm{C}$ to retrieve the antigen. After washing in PBS, the sections were blocked in a blocking solution (using $\% 2$ bovine serum albumin+\%5 normal goat serum) for non-specific binding for 40 minutes at room temperature, and subsequently incubated overnight at $4^{\circ} \mathrm{C}$ with primary rabbit anti-ly6G monoclonal antibody (1:250). On the following day, sections were then washed twice with PBS and incubated with Alexa-flour 568 goat anti-rabbit antibody (1:600) for 1 hour in a dark room. Finally, the sections were rinsed and mounted using antifade medium, and then examined using the Olympus fluorescence microscope (Olympus, Tokyo, Japan).

Macrophage infiltration and astrogliosis were evaluated respectively by the expression of the CD68 (ED1) and glial fibrillary acidic protein (GFAP) at 72 hours and 4 weeks after surgery in the sham injured and SCI groups. Samples were prepared under the same condition as previously described for Ly6G antibody with the difference that we applied permeabilization with triton $\times 100$. Rabbit anti-CD68 and rabbit anti-GFAP primary antibodies (1:200; Abcam, Berlin, Germany), were detected by goat anti-rabbit-FITC and goat antirabbit-Alexa flour 568 (1:600; Abcam) secondary antibodies, respectively.

\section{Evaluation of locomotor activity}

To evaluate the outcome of the new therapeutic intervention, we evaluated the motor function of the hind limbs pre-operatively, one day after SCI, and then every week for 4 weeks using the Basso mouse scale (BMS) in all experimental groups. Mice were allowed to step spontaneously in an open field and their behavioral patterns such as movements of the hip, knee and ankle joints, weight support and stepping, coordination, paw position, and trunk stability were accurately observed by two individuals and scored ranging from 0 indicating complete paralysis to 9 indicating normal movement of the hind limb and recorded the average score of left and right hind limbs as BMS score [24]. BMS scores were used to evaluate and compare motor function recovery in different groups over a period of 4 weeks after injury.

\section{Assessment of lesion volume}

Four weeks post-injury, we estimated the spinal cord lesion volume in all experimental groups. After deparaffinization through xylenes, and rehydration in graded alcohols, the serial cross sections were stained with cresyl violet. For systematic random sampling, one of every three cross section were selected. The lesion volume was estimated by the stereological counting tools on a Nikon E-200 microscope (Nikon) connected to a computer on the selected sections. The lesion area, including both the cavity and surrounding spared tissue, was manually outlined in images of every section viewed on the monitor, and then the surface area was calculated. Lesion volume was assessed by Cavalieri's principle by using the formula: $V=\Sigma A$.d, where " $\Sigma A$ " is the surface area summation obtained and " $\mathrm{d}$ " represents the distance between sections [25].

\section{Statistical analyses}

Statistical analyses were done using Graph-pad Prism software (version 6.07; GraphPad Software, San Diego, CA, USA). Repeated measurement ANOVA was used for evaluation of body weight and BMS scores during different time points across different groups. For the assessments of a variable between groups in a defined time, one-way ANOVA followed by Tukey's for multiple comparisons test was used.

\section{Results}

\section{Effect of ibrutinib on body weight after spinal cord injury}

A slight weight loss occurred in all animals on the day after surgery. Subsequently, an increasing trend in body weight was observed in the control and sham groups during 4 weeks following SCI, however, ibrutinib administration immediately or 12-hour after SCI slowed this increasing trend. Conversely, double injection of ibrutinib induced weight loss so that the body weight in this group was significantly lower than that of the control and sham groups after 4 weeks $(P<0.05$; Fig. 2).

\section{Evaluation of neutrophil infiltration after spinal cord injury using morphological criteria and immunofluorescent staining against Ly6G}

Regarding neutrophil infiltration into the injured parenchyma following SCI, statistical analysis revealed that the immediate administration of ibrutinib significantly decreased this index compared to the control group $(P<0.01)$. In addition, the injection of ibrutinib 12-hour after SCI suggested a similar effect $(P<0.01)$. Notably, neutrophil infiltration dramatically dropped in the group received ibrutinib both immediately and 12-hour after SCI $(P<0.001$; 
A


C
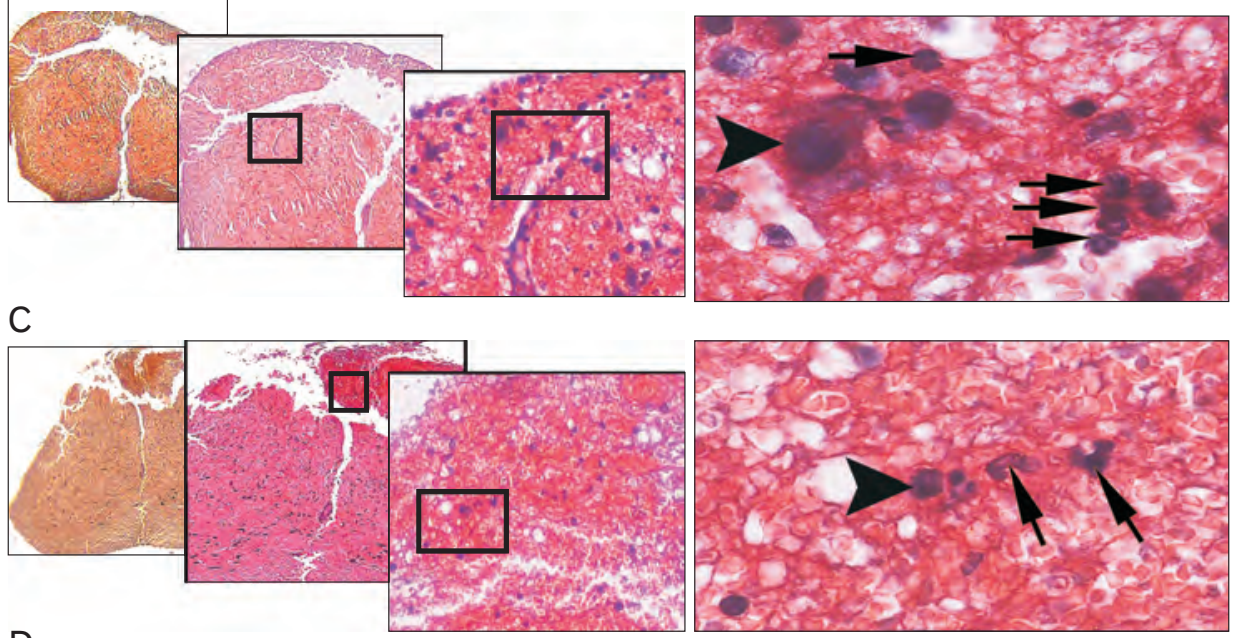

D

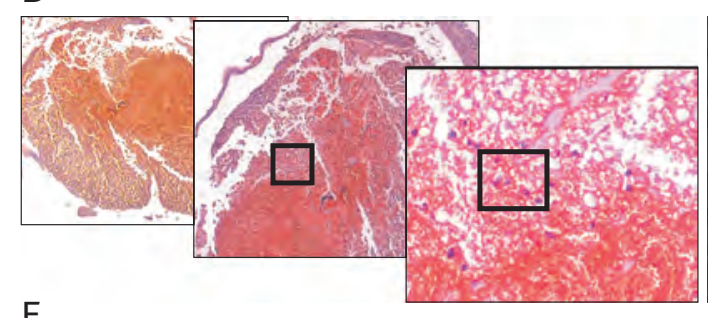

E
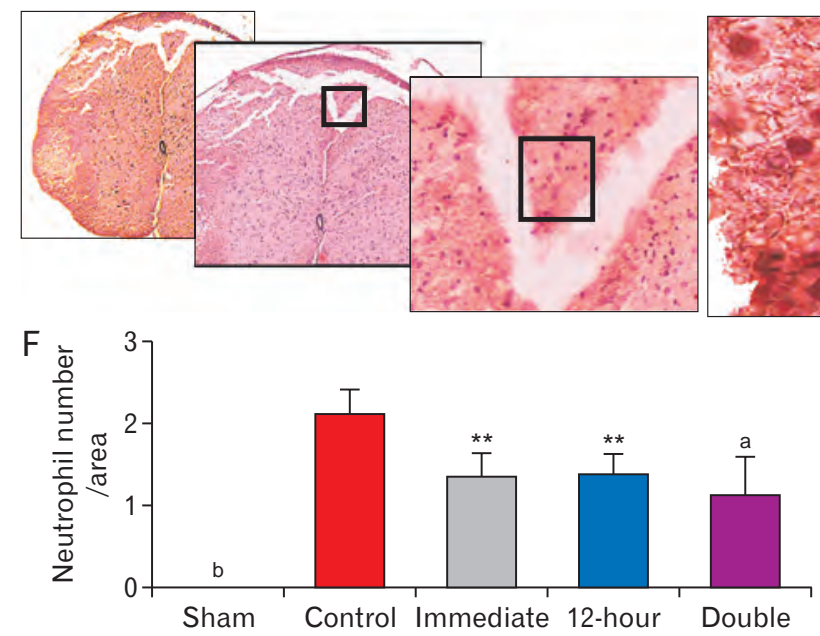

Fig. 3. Neutrophil infiltration analysis 24hour after SCI: ibrutinib administration significantly decreased neutrophil influx into the injured neural tissue after SCI. Representative images show the infiltrated polymorphonuclear neutrophils at 24 hours post-injury in each group: (A) Sham, (B) Control, (C) Ibrutinib/ Immediate, (D) Ibrutinib/12-hour, (E) Ibrutinib/double injection. Neutrophils are visualized as cells with annular and multi-lobulated nuclei (arrows), whereas neuronal cells are distinguished by their single round nuclei (arrowheads). (F) Graph showing mean neutrophil number per field of view. Congo redHematoxylin staining. Low to high magnification: $4 \times, 10 \times, 20 \times, 60 \times$ objective lens respectively. SCI, spinal cord injury. ${ }^{*} P<0.01,{ }^{a} P<0.001$ vs. $S C I /$ vehicle, ${ }^{\mathrm{b}} P<0.0001$ sham vs. other groups.
Fig. 3). Laminectomy alone did not evoke neutrophil infiltration in the spinal cord of the sham injured animals. Furthermore, investigation of Ly6G positive cells as a surface marker of neutrophils confirmed that a significant neutro- phil depletion occurred in all ibrutinib treated groups 24 hours after SCI (Fig. 4). 



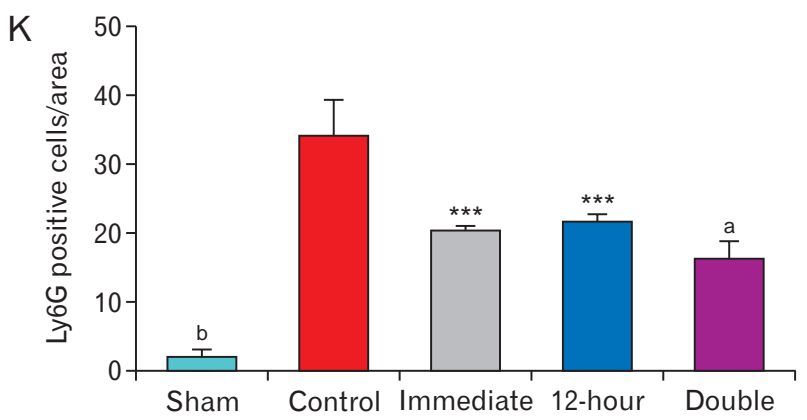

Fig. 4. Immunofluorescence based detection of Ly6G positive cells in the lesion site 24 hours after spinal cord injury (SCI): ibrutinib administration significantly decreased Ly6G positive cells in the lesion site 24 hours post-injury. Representative images are provided at the same levels in different groups: (A, B) Sham, (C, D) Control, (E, F) Ibrutinib/Immediate, (G, H) Ibrutinib/12-hour, (I, J) Ibrutinib/double injection, (K) Graph showing mean Ly6G positive cells per field of view. Magnification: upper panel at $4 \times$ and lower panel at $100 \times$. Asterisks represent Ly6G positive cells, immunolabeled with red fluorescent Alexa-flour 568 anti-rabbit antibody. ${ }^{* \star *} P<0.001,{ }^{\mathrm{a}} P<0.0001$ vs. $S C I /$ vehicle, ${ }^{\mathrm{b}} P<0.0001$ sham vs. other groups.

\section{Macrophage infiltration and reactive astrogliosis around the lesion epicenter after spinal cord injury}

Immunofluorescent staining against ED1 (CD63) and GFAP at 72 hours and 4 weeks after injury has confirmed the presence of cord injury in the SCI animals. While few ED1 immunoreactive cells could be detected in the spinal cord of the sham injured animals, many ED1 positive macrophages and activated resident microglial cells were scattered throughout the lesion site of the SCI animals (Fig. 5A-D). Similarly, after 4 weeks post-surgery, astrocytes expressing GFAP were scattered throughout the spinal cord in the sham injury group whereas in the SCI group patches of overexpressing GFAP positive astrocytes could be visualized around the lesion epicenter in the vehicle treated SCI animals further confirming the presence of cord injury in the SCI group (Fig. 5E-H).

\section{Effect of ibrutinib on Basso mouse scale score after spinal cord injury}

Hind-limb motor function improvement was evaluated in injured mice in an open field at one day, 1-week, 2-week, 3-week, and 4-week after injury, and their BMS scores were measured. Preoperatively, all of the mice showed normal gait with a $9.0 \pm 0.00$ score whereas, after the surgery, the BMS score dropped to $0.0 \pm 0.5$ in SCI group confirming a complete motor paralysis on day one. In contrast, the BMS score didn't change in sham injured animals. Over four weeks, immediate and double-injection groups considerably showed a trend of increase in BMS scores that become significantly different compared to the control (vehicle treatment) group $(P<0.01)$. Also, a moderate but significant increase in the BMS score was achieved in the 12-hour treatment group $(P<0.05$ compared to the control group). Furthermore, statistical analysis indicated that the BMS score in the control group reached a plateau by the fourth week; however, the trend of recovery in 


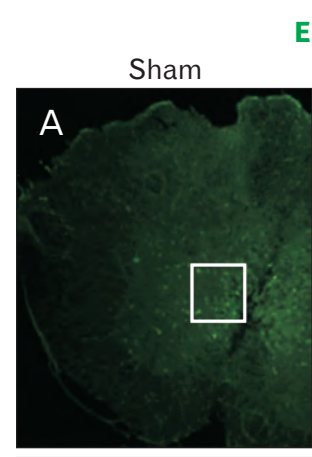

EDI
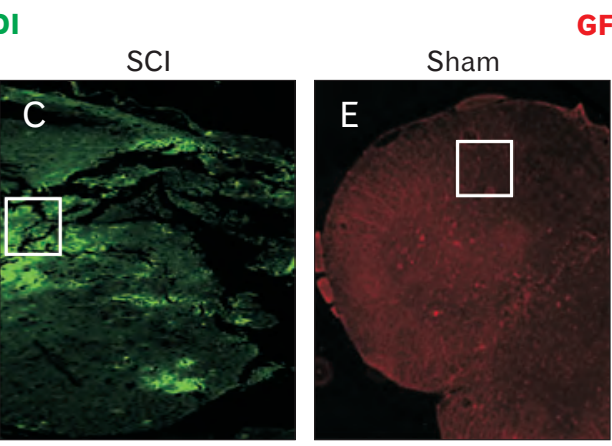

GFAP
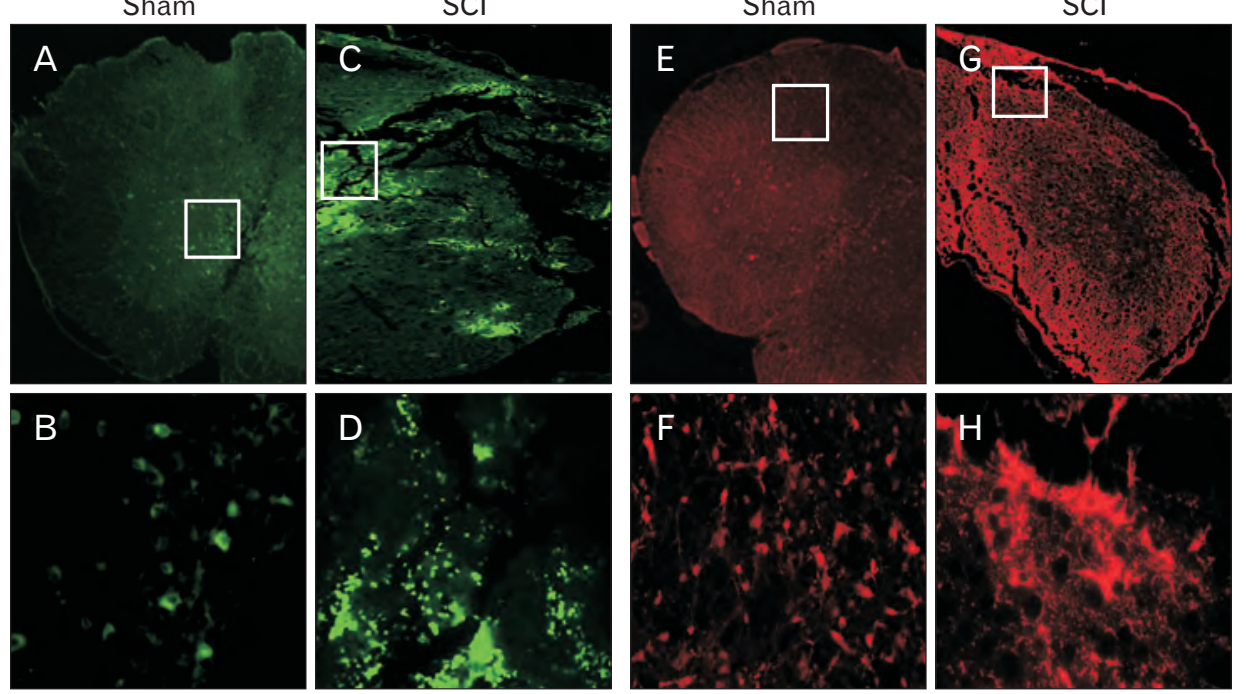

Fig. 5. Morphological confirmation of injury in the spinal cord: to confirm injury in the spinal cord, ED1 (CD68) (A-D) and glial fibrillary acidic protein (GFAP) staining (E-H) were performed respectively at 72 hours and 4 weeks after surgery. While ED1 and GFAP are respectively expressed by some resident microglial cells (A, B) and the astrocytes (E, F) in the sham injured cord tissue, their number, and staining intensity increased in the injured cord tissue due to the activation of microglial cells, influx of macrophages (C, D) and reactive astrogliosis (G, H). Magnification: upper panel at $4 \times$ and lower panel at $40 \times$. SCI, spinal cord injury.

the ibrutinib treated groups was on the rise (Fig. 6).

\section{Effect of ibrutinib on lesion volume after spinal cord injury}

With respect to the size of the lesion following SCI, statistical analysis demonstrated that administration of ibrutinib immediately after surgery significantly reduced this index compared to the control (vehicle treatment) group $(P<0.001)$. Similarly, the lesion volume was decreased in the group that received ibrutinib 12 hours after SCI $(P<0.001)$. Moreover, injection of ibrutinib both immediately and 12 hours after SCI also resulted in a considerable reduction in lesion volume $(P<0.001$, Fig. 7$)$.

\section{Discussion}

Herein, we found that the administration of ibrutinib, a selective BTK inhibitor, in mouse contusion model of acute SCI would reduce neural tissue damage and improve locomotor function. In the histological analysis of neutrophils using Congo red-Hematoxylin staining, we demonstrated that ibrutinib administration at the first 12 hours after the onset of SCI would result in a decreased neutrophil influx into the injured spinal cord tissue. This effect was more profound when ibrutinib was used both immediately and

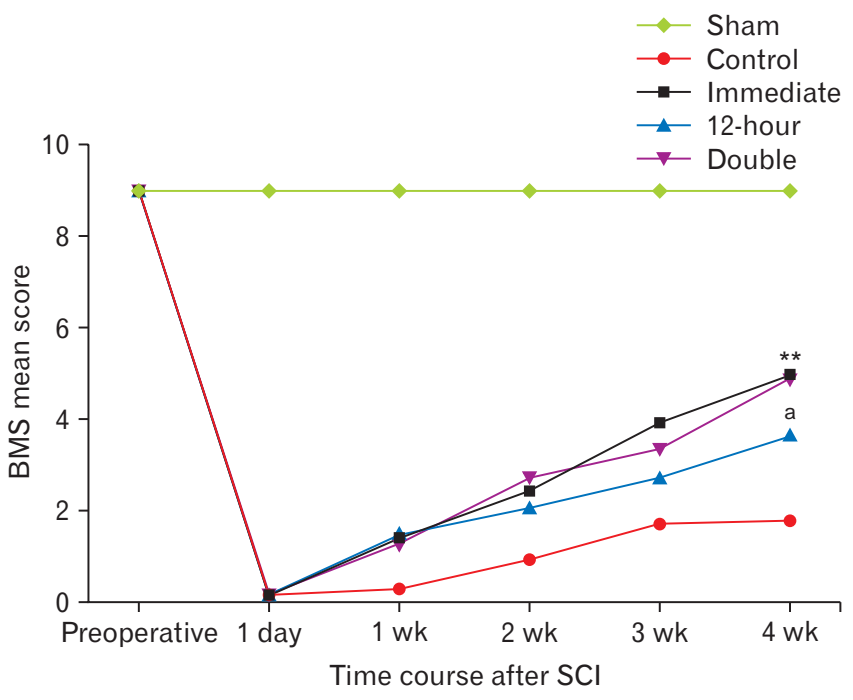

Fig. 6. Hind limb locomotor function after SCI: Ibrutinib administration significantly improved locomotor recovery. The BMS score was estimated pre-operatively, on the first day and every week to assess behavioral recovery following SCI. SCI, spinal cord injury; BMS, Basso mouse scale. ${ }^{*} P<0.01,{ }^{a} P<0.05$ vs. control.

12-hour after the induction of SCI. In addition, our complementary immunofluorescent study proved the same trend of decrease in the number of Ly6G-positive cells in ibrutinib treated groups, although the number of Ly6G-positive cells was much higher than the number of neutrophils in Congo 

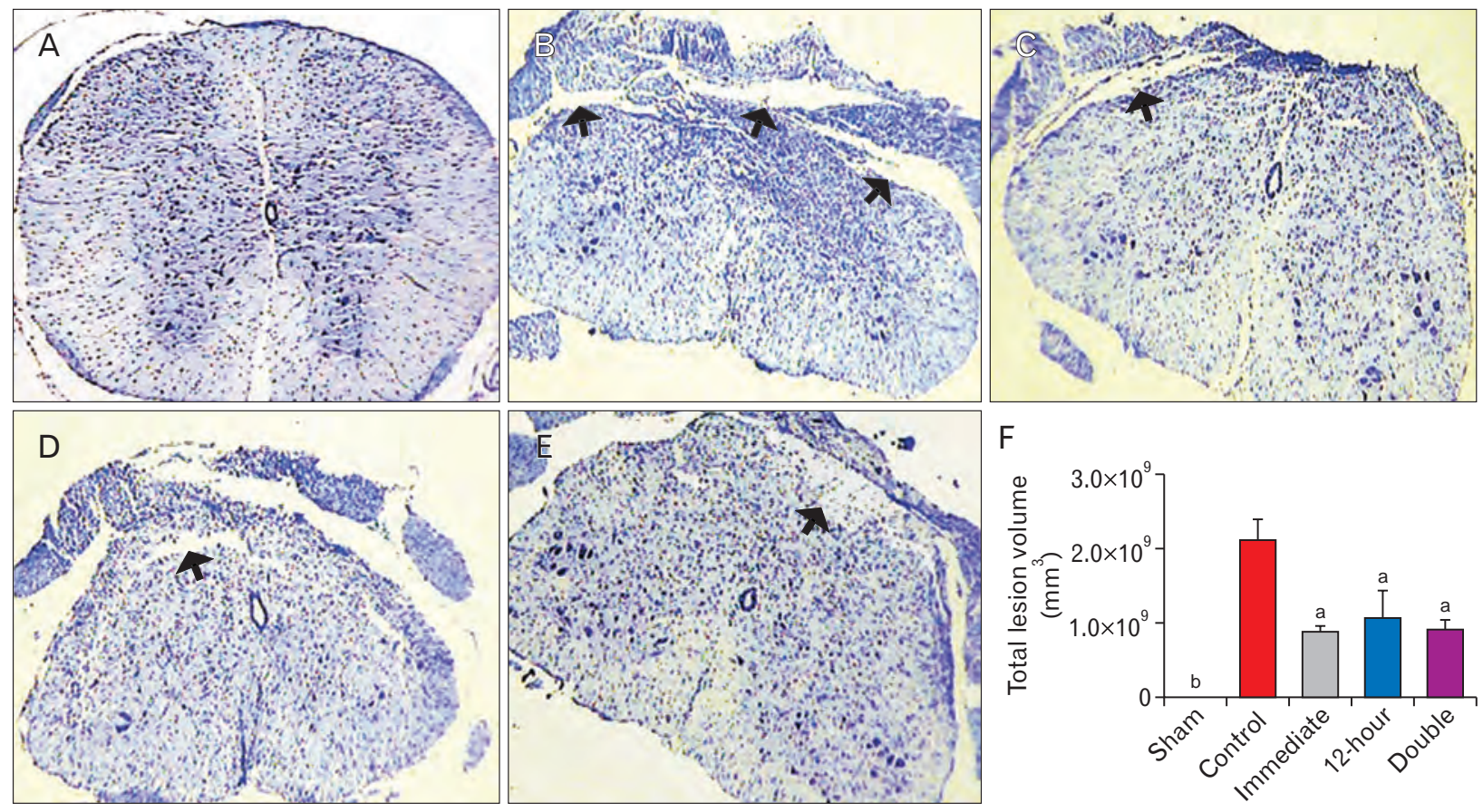

Fig. 7. Total lesion volume after SCI: ibrutinib administration significantly diminished the lesion volume four weeks after SCI. Cavities (arrows) and the surrounding spared tissue were measured in the different groups by Cavalieri's principle. (A) Sham, (B) Control, (C) Ibrutinib/ Immediate, (D) Ibrutinib/12-hour, (E) Ibrutinib/double injection. (F) Graph showing total lesion volume in different treatment groups. Cresyl violet staining; magnification: $90 \times .{ }^{\mathrm{a}} P<0.001$ vs. control, ${ }^{\mathrm{b}} \mathrm{P}<0.001$ vs. other groups.

red-Hematoxylin histological preparations. This discrepancy could be justified by the fact that Ly6G is expressed in neutrophils and other myeloid cells [26, 27], thus the presence of other innate immune cells such as monocytes and other leukocytes in the lesion site cannot be ruled out without using a comprehensive library of antibodies that were not available for this study.

Moreover, our study displayed a considerable reduction in the lesion volume and, most importantly, an improved locomotor behavioral recovery of the hind limbs after ibrutinib administration in all treated groups. However, statistical analysis unveiled a higher recovery rate in the immediate and double injection treatment groups during the four weeks following SCI. Although double injection of ibrutinib resulted in better neurological outcomes, it significantly decreased animals' body weight compared to the control group. Importantly, we also observed accumulation of pus surrounding the surgical area and fluid in the abdomen of some animals in the ibrutinib double injection group in early days after SCI whereas no similar conditions were seen in control and other treatment groups.

Following the primary SCI, the first leukocyte population proceeds into the injured site are polymorphonuclear neutrophils. Excessive neutrophil recruitment may exacerbate the injury process by damaging the neural parenchyma, and by releasing the toxic effectors in the acute phase [7]. In this study, we governed exaggerated inflammatory response post SCI by using an FDA-approved chemotherapy drug called ibrutinib, which irreversibly blocks a type of kinase called BTK, a small molecule in the signaling pathway of immune cells such as B cells and myeloid cells $[9,28]$. Here, our findings established that ibrutinib treatment within the acute phase limited the neutrophil quantity 24 hours following SCI, and thereby attenuated the extent of tissue damage 4 weeks later, hence provided functional improvement. In this study, because the drug was administered in the acute phase of the injury, the effect of the drug on mononuclear leukocytes such as B cells that enter the chronic phase can be neglected.

Of note, we compared the results obtained from different injections and introduced the most effective time point. Body weight loss and wound infection following twice administration of ibrutinib suggest that this pattern of injection is not safe compared to single-dose injection. Our findings 
are in agreement with the study where exposure to ibrutinib impairs the anti-fungal innate immunity both in vitro and in vivo [29]. Furthermore, it was shown that the risk of fungal infections had increased in chronic lymphocytic leukemia patients received ibrutinib [30,31].

Emerging studies have clarified downstream signaling pathways in myeloid cells where BTK has a crucial role in their activation. These studies have revealed that BTK regulates the activation of a tissue-damage sensor called NLRP3 (NLR family, pyrin domain containing 3) inflammasome or stimulates the cleavage of caspase-1 which are necessary for the conversion of the inactive pro-IL-1 $\beta$, to the mature and active form that is released from myeloid cells to provoke an inflammatory response $[16,32]$. The involvement of BTK in the recruitment of myeloid cells into the damaged area suggested that BTK inhibitor may impede trans-endothelial migration which is of prime importance in inflammatory responses $[16,33]$. Other studies manifested that BTK contributes to a form of cell death termed pyroptosis, the event which triggers the recruitment of more neutrophils to the lesion site $[34,35]$. Ibrutinib with inhibitory potency on BTK blocks signaling cascade in myeloid cells through covalently binding to the cysteine amino acid of this kinase [36]. Our study showed that ibrutinib treatment lowers the number neutrophils and Ly6G-positive cells in the lesion site but does not completely deplete them. Therefore, limiting the number of neutrophils in the lesion site at the first 24 hours post injury reduces the severity of destructive events triggered by the neutrophils and at same time the presence of limited number of neutrophils in the early stages of injury will help resolving inflammation as have been reported by others [37]. In agreement with this notion, Stirling et al. [38] showed that depletion of the early arriving neutrophils using an anti-Ly6G/ Gr-1 antibody after SCI, would stop restoration events and result in severe neurological outcomes and increasing the lesion volume. Conversely, as shown by Ito et al. [18], ibrutinib treatment after ischemic stroke did not change the number of neutrophils and macrophages in the lesion site but instead impaired NLRP3 activation through BTK inhibition which in turn suppressed maturation of IL-1 $\beta$ by the infiltrating neutrophils and macrophages resulting in reduced infarct volume, and improved neurological deficits. Although using a similar dose of ibrutinib in the first 24 hours post injury, the discrepancy between Ito's and ours study regarding the effect of ibrutinib on the number of neutrophil and macrophages in the lesion area could mainly be attributed to the nature of injury models (ischemic stroke vs contusive SCI). Although we did not look for BTK signaling and downstream pathways in our study, it is also expected to occur when ibrutinib used in SCI model and hence diminished the extent of inflammation and resulted in tissue sparing and functional recovery. Further studies are required to mechanistically address this difference.

Overall, our findings in the context of similar studies evaluating the effects of BTK-inhibitors on acute inflammatory conditions such as in patients with respiratory adverse events $[12,19]$ and patients with COVID-19 [39] and animal model of liver ischemia-reperfusion [17] demonstrated that ibrutinib treatment has a positive correlation with spinal cord preservation and functional recovery. Some researchers have demonstrated that ibrutinib has a favorable tolerability and safety profile [40]. For years, other medications including glucocorticoids and nonsteroidal anti-inflammatory drugs (NSAIDs) were used to manage secondary events after SCI. However, these medications showed a wide range of potential complications such as gastrointestinal ulcer/bleeding and increased risks for respiratory, urinary tract, and wound infections, hyperglycemia, steroid induced myopathy, and mortality [8, 41-44]. Ibrutinib therapy seems to act as a safe treatment with fewer side effects that could be used to lessen complications associated with the SCI.

In conclusion, the present study, for the first time, implicates ibrutinib as a positive regulator of inflammation after SCI. We demonstrated that systemic ibrutinib administration in the acute phase, through downregulation of excessive neutrophil recruitment to the lesion site can be considered as a promising approach for treating SCI. With a significant motor recovery in all ibrutinib administration paradigms comparing to the untreated control condition and less body weight loss and wound infection in the single injection paradigms (immediately and 12 hours after SCI), immediate administration of a single dose of ibrutinib in the first 24 hours after SCI is recommended to reduce neutrophil infiltration into the injured cord, thus minimizing inflammation and enhancing locomotor recovery.

\section{ORCID}

Somayyeh Torabi: https://orcid.org/0000-0002-5305-9773

Seyed Hadi Anjamrooz:

https://orcid.org/0000-0002-6200-6606

Zahra Zeraatpisheh: 
https://orcid.org/0000-0002-6153-3551

Hadi Aligholi: https://orcid.org/0000-0002-2241-5296

Hassan Azari: https://orcid.org/0000-0002-8021-3166

\section{Author Contributions}

Conceptualization: ST, HA, HA, SHA. Data acquisition: ST, ZZ. Data analysis or interpretation: ST, HA, HA, SHA. Drafting of the manuscript: ST, HA, HA. Critical revision of the manuscript: HA, HA, SHA. Approval of the final version of the manuscript: all authors.

\section{Conflicts of Interest}

No potential conflict of interest relevant to this article was reported.

\section{Acknowledgements}

This work was supported by the Shiraz University of Medical Sciences (Grant no. 1397-04-11-15704, 2018).

\section{References}

1. Lim PA, Tow AM. Recovery and regeneration after spinal cord injury: a review and summary of recent literature. Ann Acad Med Singap 2007;36:49-57.

2. Abbaszadeh HA, Niknazar S, Darabi S, Ahmady Roozbahany N, Noori-Zadeh A, Ghoreishi SK, Khoramgah MS, Sadeghi Y. Stem cell transplantation and functional recovery after spinal cord injury: a systematic review and meta-analysis. Anat Cell Biol 2018;51:180-8.

3. Cristante AF, Barros Filho TE, Marcon RM, Letaif OB, Rocha ID. Therapeutic approaches for spinal cord injury. Clinics (Sao Paulo) 2012;67:1219-24.

4. Thuret S, Moon LD, Gage FH. Therapeutic interventions after spinal cord injury. Nat Rev Neurosci 2006;7:628-43.

5. Kwiecien JM, Dabrowski W, Dąbrowska-Bouta B, Sulkowski G, Oakden W, Kwiecien-Delaney CJ, Yaron JR, Zhang L, Schutz L, Marzec-Kotarska B, Stanisz GJ, Karis JP, Struzynska L, Lucas AR. Prolonged inflammation leads to ongoing damage after spinal cord injury. PLoS One 2020;15:e0226584.

6. Fleming JC, Norenberg MD, Ramsay DA, Dekaban GA, Marcillo AE, Saenz AD, Pasquale-Styles M, Dietrich WD, Weaver LC. The cellular inflammatory response in human spinal cords after injury. Brain 2006;129(Pt 12):3249-69.

7. Noble LJ, Donovan F, Igarashi T, Goussev S, Werb Z. Matrix metalloproteinases limit functional recovery after spinal cord injury by modulation of early vascular events. J Neurosci 2002;22:7526-35.
8. Singh PL, Agarwal N, Barrese JC, Heary RF. Current therapeutic strategies for inflammation following traumatic spinal cord injury. Neural Regen Res 2012;7:1812-21.

9. Zhou H, Hu P, Yan X, Zhang Y, Shi W. Ibrutinib in chronic lymphocytic leukemia: clinical applications, drug resistance, and prospects. Onco Targets Ther 2020;13:4877-92.

10. Campbell R, Chong G, Hawkes EA. Novel indications for Bruton's tyrosine kinase inhibitors, beyond hematological malignancies. J Clin Med 2018;7:62.

11. Tobinai K, Ogura M, Ishizawa K, Suzuki T, Munakata W, Uchida T, Aoki T, Morishita T, Ushijima Y, Takahara S. Safety and tolerability of ibrutinib monotherapy in Japanese patients with relapsed/refractory B cell malignancies. Int J Hematol 2016;103:86-94.

12. Krupa A, Fol M, Rahman M, Stokes KY, Florence JM, Leskov IL, Khoretonenko MV, Matthay MA, Liu KD, Calfee CS, Tvinnereim A, Rosenfield GR, Kurdowska AK. Silencing Bruton's tyrosine kinase in alveolar neutrophils protects mice from LPS/immune complex-induced acute lung injury. Am J Physiol Lung Cell Mol Physiol 2014;307:L435-48.

13. Hamasy A, Wang Q, Blomberg KE, Mohammad DK, Yu L, Vihinen M, Berglöf A, Smith CI. Substitution scanning identifies a novel, catalytically active ibrutinib-resistant BTK cysteine 481 to threonine (C481T) variant. Leukemia 2017;31:177-85.

14. da Cunha-Bang C, Niemann CU. Targeting Bruton's tyrosine kinase across B-cell malignancies. Drugs 2018;78:1653-63.

15. de Porto AP, Liu Z, de Beer R, Florquin S, de Boer OJ, Hendriks RW, van der Poll T, de Vos AF. Btk inhibitor ibrutinib reduces inflammatory myeloid cell responses in the lung during murine pneumococcal pneumonia. Mol Med 2019;25:3.

16. Block H, Zarbock A. The role of the tec kinase Bruton's tyrosine kinase (Btk) in leukocyte recruitment. Int Rev Immunol 2012;31:104-18.

17. Palumbo T, Nakamura K, Lassman C, Kidani Y, Bensinger SJ, Busuttil R, Kupiec-Weglinski J, Zarrinpar A. Bruton tyrosine kinase inhibition attenuates liver damage in a mouse warm ischemia and reperfusion model. Transplantation 2017;101:32231.

18. Ito M, Shichita T, Okada M, Komine R, Noguchi Y, Yoshimura A, Morita R. Bruton's tyrosine kinase is essential for NLRP3 inflammasome activation and contributes to ischaemic brain injury. Nat Commun 2015;6:7360.

19. Liu X, Zhang J, Han W, Wang Y, Liu Y, Zhang Y, Zhou D, Xiang L. Inhibition of BTK protects lungs from trauma-hemorrhagic shock-induced injury in rats. Mol Med Rep 2017;16:192-200.

20. Florence JM, Krupa A, Booshehri LM, Davis SA, Matthay MA, Kurdowska AK. Inhibiting Bruton's tyrosine kinase rescues mice from lethal influenza-induced acute lung injury. Am J Physiol Lung Cell Mol Physiol 2018;315:L52-8.

21. Zeraatpisheh Z, Mirzaei E, Nami M, Alipour H, Ghasemian S, Azari $\mathrm{H}$, Aligholi $\mathrm{H}$. A new and simple method for spinal cord injury induction in mice. Basic Clin Neurosci. Forthcoming 2020.

22. Unal B, Kaplan S, Odaci E, Aslan H, Aksak S, Unal D, Al- 
tunkaynak BZ, Gundogdu C, Gokyar A. Neuroprotective effects of methylprednisolone and hypothermia after experimental spinal cord injury: a histopathological and stereological study. Eurasian J Med 2009;41:169-74.

23. O'Connell KE, Mikkola AM, Stepanek AM, Vernet A, Hall CD, Sun CC, Yildirim E, Staropoli JF, Lee JT, Brown DE. Practical murine hematopathology: a comparative review and implications for research. Comp Med 2015;65:96-113.

24. Basso DM, Fisher LC, Anderson AJ, Jakeman LB, McTigue DM, Popovich PG. Basso Mouse Scale for locomotion detects differences in recovery after spinal cord injury in five common mouse strains. J Neurotrauma 2006;23:635-59.

25. Jakeman LB. Assessment of lesion and tissue sparing volumes following spinal cord injury. In: Chen J, Xu XM, Xu ZC, Zhang JH, editors. Animal Models of Acute Neurological Injuries II. New York: Humana Press; 2012. p.417-42.

26. Lee SM, Rosen S, Weinstein P, van Rooijen N, NobleHaeusslein LJ. Prevention of both neutrophil and monocyte recruitment promotes recovery after spinal cord injury. J Neurotrauma 2011;28:1893-907.

27. Hawthorne AL, Popovich PG. Emerging concepts in myeloid cell biology after spinal cord injury. Neurotherapeutics 2011;8:252-61.

28. Hendriks RW, Yuvaraj S, Kil LP. Targeting Bruton's tyrosine kinase in B cell malignancies. Nat Rev Cancer 2014;14:219-32.

29. Blez D, Blaize M, Soussain C, Boissonnas A, MeghraouiKheddar A, Menezes N, Portalier A, Combadière C, Leblond V, Ghez D, Fekkar A. Ibrutinib induces multiple functional defects in the neutrophil response against Aspergillus fumigatus. Haematologica 2020;105:478-89.

30. Facchinelli D, Marchesini G, Nadali G, Pagano L. Invasive fungal infections in patients with chronic lymphoproliferative disorders in the era of target drugs. Mediterr J Hematol Infect Dis 2018;10:e2018063.

31. Rogers K. Ibrutinib and fungus: an invasive concern. Blood 2018;131:1882-4.

32. Weber ANR, Bittner Z, Liu X, Dang TM, Radsak MP, Brunner C. Bruton's tyrosine kinase: an emerging key player in innate immunity. Front Immunol 2017;8:1454.

33. Stadler N, Hasibeder A, Lopez PA, Teschner D, Desuki A, Kriege O, Weber ANR, Schulz C, Michel C, He $\beta$ G, Radsak MP. The Bruton tyrosine kinase inhibitor ibrutinib abrogates triggering receptor on myeloid cells 1-mediated neutrophil ac- tivation. Haematologica 2017;102:e191-4.

34. Miao EA, Rajan JV, Aderem A. Caspase-1-induced pyroptotic cell death. Immunol Rev 2011;243:206-14.

35. Dinarello CA. Overview of the IL-1 family in innate inflammation and acquired immunity. Immunol Rev 2018;281:8-27.

36. Das D, Hong J. Irreversible kinase inhibitors targeting cysteine residues and their applications in cancer therapy. Mini Rev Med Chem 2020;20:1732-53.

37. Neirinckx V, Coste C, Franzen R, Gothot A, Rogister B, Wislet $S$. Neutrophil contribution to spinal cord injury and repair. J Neuroinflammation 2014;11:150.

38. Stirling DP, Liu S, Kubes P, Yong VW. Depletion of Ly6G/Gr-1 leukocytes after spinal cord injury in mice alters wound healing and worsens neurological outcome. J Neurosci 2009;29:75364.

39. Treon SP, Castillo JJ, Skarbnik AP, Soumerai JD, Ghobrial IM, Guerrera ML, Meid K, Yang G. The BTK inhibitor ibrutinib may protect against pulmonary injury in COVID-19-infected patients. Blood 2020;135:1912-5.

40. Nastoupil LJ, Lunning MA, Vose JM, Schreeder MT, Siddiqi T, Flowers CR, Cohen JB, Burger JA, Wierda WG, O'Brien S, Sportelli P, Miskin HP, Purdom MA, Weiss MS, Fowler NH. Tolerability and activity of ublituximab, umbralisib, and ibrutinib in patients with chronic lymphocytic leukaemia and nonHodgkin lymphoma: a phase 1 dose escalation and expansion trial. Lancet Haematol 2019;6:e100-9.

41. Choi SH, Sung CH, Heo DR, Jeong SY, Kang CN. Incidence of acute spinal cord injury and associated complications of methylprednisolone therapy: a national population-based study in South Korea. Spinal Cord 2020;58:232-7.

42. Cheung V, Hoshide R, Bansal V, Kasper E, Chen CC. Methylprednisolone in the management of spinal cord injuries: lessons from randomized, controlled trials. Surg Neurol Int 2015;6:142.

43. Chikuda H, Yasunaga H, Takeshita K, Horiguchi H, Kawaguchi H, Ohe K, Fushimi K, Tanaka S. Mortality and morbidity after high-dose methylprednisolone treatment in patients with acute cervical spinal cord injury: a propensity-matched analysis using a nationwide administrative database. Emerg Med J 2014;31:201-6.

44. Suberviola B, González-Castro A, Llorca J, Ortiz-Melón F, Miñambres E. Early complications of high-dose methylprednisolone in acute spinal cord injury patients. Injury 2008;39:748-52. 